\title{
Spherical Images of W-Direction Curves in Euclidean 3-Space
}

\author{
Ìlkay Arslan Güven ${ }^{1}$, Semra Kaya Nurkan ${ }^{2} \&$ Ìpek Ağaoğlu Tor ${ }^{1}$ \\ ${ }^{1}$ Department of Mathematics, Faculty of Arts and Science, Gaziantep University, TR-27310 Gaziantep, Turkey \\ 2 Department of Mathematics, Faculty of Arts and Science, Usak University, TR-64200 Usak, Turkey \\ Correspondence: Ìlkay Arslan Güven, Department of Mathematics, Faculty of Arts and Science, Gaziantep University, \\ TR-27310 Gaziantep, Turkey
}

Received: March 12, 2020 Accepted: April 23, 2020 Online Published: May 6, 2020

doi:10.5539/jmr.v12n3p39 URL: https://doi.org/10.5539/jmr.v12n3p39

\begin{abstract}
In this paper, we study the spherical indicatrices of W-direction curves in three dimensional Euclidean space which were defined by using the unit Darboux vector field $W$ of a Frenet curve. We obtain the Frenet apparatus of these spherical indicatrices and the characterizations of being general helix and slant helix. Moreover we give some properties between the spherical indicatrices and their associated curves.
\end{abstract}

2000 Mathematics Subject Classification: 53A04, 14H50

Keywords: W-direction curve, spherical indicatrix, general helix

\section{Introduction}

The theory of curves is a subbranch of geometry which deals with curves in Euclidean space or other spaces by using differential and integral calculus. One of the most studied topic in curve theory is associated curves like involute-evolute pairs, Bertrand curve pairs, Mannheim partner curves and W-direction curves. Working with these associated curves is nice aspect that these curves can be characterizated by the properties and behavior of the main curves of them.

The most commonly used ones to characterize curves are general or cylindrical helix and slant helix. A general helix in $\mathbb{E}^{3}$ is defined as: its tangent vector field makes a constant angle with a fixed direction. If the principal normal vector field makes a constant angle with a fixed direction, it is called slant helix. Izumiya and Takeuchi founded that a curve is a slant helix if and only if the geodesic curvature of the principal image of the principal normal indicatrix which is

$$
\delta(s)=\left(\frac{\kappa^{2}}{\left(\kappa^{2}+\tau^{2}\right)^{\frac{3}{2}}} \cdot\left(\frac{\tau}{\kappa}\right)^{\prime}\right)(s)
$$

is a constant function (Izumiya \& Takenchi, 2004).

Choi and Kim (2012) created the principal(binormal)-direction curves and principal(binormal)-donor curve of a Frenet curve in $\mathbb{E}^{3}$. They gave the relation of curvature and torsion between the principal-direction curve and its mate curve. They also defined a new curve called PD-rectifying curve and gave a new characterization of a Bertrand curve by means of the PD-rectifying curve. They made an application of associated curves and studied a general helix and slant helix as principal-donor and second principal-donor curve of a plane, respectively. Then Choi et al. (2012). worked on the principal(binormal)-direction curve and principal(binormal)-donor curve of a Frenet non-lightlike curves in $\mathbb{E}_{1}^{3}$.

After that Körpınar et al. (2013). introduced associated curves according to Bishop frame in $\mathbb{E}^{3}$.

Recently, Macit and Düldül (2014) defined W-direction curve, W-rectifying curve and V-direction curve of a Frenet curve in $\mathbb{E}^{3}$ and also principal-direction curve, $B_{1}$-direction curve, $B_{2}$-direction curve and $B_{2}$-rectifying curve in $\mathbb{E}^{4}$, where $B_{1}$ is the first binormal vector field and $B_{2}$ is the second binormal vector field of the Frenet frame of a curve in $\mathbb{E}^{4}$. These curves were given as the integral curves of vector fields taken from the Frenet frame or Darboux frame along a curve. They gave the relationship of the Frenet vector fields, the curvature and the torsion between the associated curves and their main curve.

When all unit Frenet vectors of a curve are moved to the center of the unit sphere $S^{2}$, their end points describe different curves on the sphere. These described curves are called spherical indicatrices. For unit tangent vector, unit principal normal vector and unit binormal vector, the names of the spherical indicatrices are tangent indicatrix, principal normal indicatrix and binormal indicatrix, respectively. If any of the Frenet vectors is $X$ of a curve given with the arc-lenght 
parameter $s$, then the equation of the spherical indicatrix is given by

$$
\beta\left(s^{*}\right)=X(s) .
$$

where $s^{*}$ is the arc-length parameter of spherical indicatrix (Struik, 1957).

There are many works on spherical indicatrices. Kula and Yaylı (2005), investigated spherical images of tangent and binormal indicatrix of a slant helix. They found that the spherical images are spherical helices.

Kula et al. (2010) gave some characterizations for a unit speed curve in $\mathbb{R}^{3}$ to being a slant helix by using its tangent, principal normal and binormal indicatrix.

Tunçer and Ünal (2012) studied spherical indicatrices of a Bertrand curve and its mate curve. They obtained relations between spherical images and new representations of spherical indicatrices.

In this paper, we study the spherical indicatrices of W-direction curves. We obtain the Frenet apparatus of tangent indicatrix and binormal indicatrix via Frenet vector fields, curvature and torsion of the main curves. We give the characterizations of being general helix and slant helix in terms of these image curves.

\section{Preliminaries}

Let $\beta: I \longrightarrow \mathbb{E}^{3}$ be a curve and $\{T, N, B\}$ denote the Frenet frame of $\beta . T(s)=\beta^{\prime}(s)$ is called the unit tangent vector of $\beta$ at $s$. $\beta$ is a unit speed curve (or parametrized by arc-length $s$ ) if and only if $\left\|\beta^{\prime}(s)\right\|=1$. The curvature of $\beta$ is given by $\kappa(s)=\left\|\beta^{\prime \prime}(s)\right\|$. The unit principal normal vector $N(s)$ of $\beta$ at $s$ is given by $\beta^{\prime \prime}(s)=\kappa(s)$. $N(s)$. Also the unit vector $B(s)=T(s) \times N(s)$ is called the unit binormal vector of $\beta$ at $s$. Then the famous Frenet formula holds as:

$$
\begin{aligned}
T^{\prime}(s) & =\kappa(s) \cdot N(s) \\
N^{\prime}(s) & =-\kappa(s) \cdot T(s)+\tau(s) B(s) \\
B^{\prime}(s) & =-\tau(s) N(s)
\end{aligned}
$$

where $\tau(s)$ is the torsion of $\beta$ at $s$ and calculated as $\tau(s)=\left\langle N^{\prime}(s), B(s)\right\rangle$ or $\tau(s)=\left\|B^{\prime}(s)\right\|$.

Also the Frenet vectors of a curve $\beta$, which is given by arc-length parameter $s$, can be calculated as:

$$
\begin{aligned}
T(s) & =\beta^{\prime}(s) \\
N(s) & =\frac{\beta^{\prime \prime}(s)}{\left\|\beta^{\prime \prime}(s)\right\|} \\
B(s) & =T(s) \times N(s) .
\end{aligned}
$$

For the unit speed curve $\beta: I \longrightarrow \mathbb{E}^{3}$, the vector

$$
W(s)=\tau(s) T(s)+\kappa(s) B(s)
$$

is called the Darboux vector of $\beta$ which is the rotation vector of trihedron of the curve with curvature $\kappa \neq 0$ when a point moves along the curve $\beta$.

A unit speed curve $\beta: I \longrightarrow \mathbb{E}^{n}$ is a Frenet curve if $\beta^{\prime \prime}(s) \neq 0$, so it has the non-zero curvature.

Definition 1. Let $\beta$ be a Frenet curve in $\mathbb{E}^{3}$ and $W$ be the unit Darboux vector field of $\beta$. The integral curve of $W(s)$ is called $W$-direction curve of $\beta$. Namely, if $\bar{\beta}$ is the $W$-direction curve of $\beta$, then $W(s)=\bar{\beta}^{\prime}(s)$, where $W=\frac{1}{\sqrt{\kappa^{2}+\tau^{2}}}(\tau T+\kappa B)$ (Macit $\mathcal{E}$ Dïldül, 2014).

Let the Frenet apparatus of a Frenet curve $\beta$ and its W-direction curve be $\{T, N, B, \kappa, \tau\}$ and $\{\bar{T}, \bar{N}, \bar{B}, \bar{\kappa}, \bar{\tau}\}$ respectively. The relations of Frenet apparatus between the main curve and W-direction curve are given in (Macit \& Düldül, 2014) as:

$$
\begin{aligned}
& \bar{T}=\frac{\tau}{\sqrt{\kappa^{2}+\tau^{2}}} T+\frac{\kappa}{\sqrt{\kappa^{2}+\tau^{2}}} B \\
& \bar{N}=-\frac{\kappa}{\sqrt{\kappa^{2}+\tau^{2}}} T+\frac{\tau}{\sqrt{\kappa^{2}+\tau^{2}}} B \\
& \bar{B}=-N \\
& \bar{\kappa}=\frac{\left|\tau \kappa^{\prime}-\tau^{\prime} \kappa\right|}{\kappa^{2}+\tau^{2}}, \quad \bar{\tau}=\sqrt{\kappa^{2}+\tau^{2}}
\end{aligned}
$$


Remark 1. In this paper we take the signs of absolute value positive. If the sign will be taken negative, the expressions similarly have the other signs.

Theorem 1. Let $\bar{\beta}$ be the $W$-direction curve of $\beta$ which is not a general helix. Then $\bar{\beta}$ is a general helix if and only if $\beta$ is a slant helix (Macit E Düldül, 2014).

Theorem 2. A curve is a general helix if and only if $\frac{\tau}{\kappa}=$ constant (O'Neill, 2006).

Let $\alpha$ be any curve of arc-length parameter $s$ and has the Frenet frame $\{T, N, B\}$ along $\alpha$. If $\alpha$ is on a surface, the frame $\{T, V, U\}$ along the curve $\alpha$ is called the Darboux frame where $T$ is the unit tangent vector of $\alpha, U$ is the unit normal of the surface and $V$ is the unit vector given by $V=U \times T$. The relations between these vectors and their derivatives are (O’Neill, 2006):

$$
\left[\begin{array}{c}
T^{\prime} \\
V^{\prime} \\
U^{\prime}
\end{array}\right]=\left[\begin{array}{ccc}
0 & \kappa_{g} & \kappa_{n} \\
-\kappa_{g} & 0 & \tau_{g} \\
-\kappa_{n} & -\tau_{g} & 0
\end{array}\right]\left[\begin{array}{c}
T \\
V \\
U
\end{array}\right]
$$

where $\kappa_{g}$ is the geodesic curvature, $\kappa_{n}$ is the normal curvature and $\tau_{g}$ is the geodesic torsion.

The geodesic curvature, normal curvature and geodesic torsion with respect to the surface are also given respectively by (O’Neill, 2006):

$$
\begin{gathered}
\kappa_{g}=\left\langle U \times T, T^{\prime}\right\rangle \\
\kappa_{n}=\left\langle\alpha^{\prime \prime}, U\right\rangle
\end{gathered}
$$

and

$$
\tau_{g}=\left\langle U \times U^{\prime}, T\right\rangle .
$$

For a curve $\alpha$ which is lying on a surface, the following statements are satisfied (O'Neill, 2006):

1) $\alpha$ is a geodesic curve if and only if the geodesic curvature of the curve with respect to the surface vanishes.

2) $\alpha$ is a asymptotic line if and only if the normal curvature of the curve with respect to the surface vanishes.

3) $\alpha$ is a principal line if and only if the geodesic torsion of the curve with respect to the surface vanishes.

\section{Spherical Images of W-direction Curves}

In this section, we will introduce tangent indicatrix and binormal indicatrix which are spherical indicatrices of W-direction curves. We find their Frenet apparatus and give some results of being general helix and slant helix.

Let $\beta$ be a curve with arc-length parameter $s$ and $\bar{\beta}$ be the W-direction curve of $\beta$. The arc-length parameter $\bar{s}$ of $\bar{\beta}$ which is an integral curve of $\beta$, can be taken as $\bar{s}=s$ (Choi \& Kim, 2012). The Frenet apparatus of $\beta$ and $\bar{\beta}$ are $\{T, N, B, \kappa, \tau\}$ and $\{\bar{T}, \bar{N}, \bar{B}, \bar{\kappa}, \bar{\tau}\}$ respectively. Here also $\delta$ is the geodesic curvature of the principal image of the principal normal indicatrix given with the equation (1.1).

Using the equation (1.2), the tangent indicatrix and binormal indicatrix of W-direction curve $\bar{\beta}$ are given with the equations

$$
\begin{aligned}
\alpha\left(s_{\alpha}\right) & =\bar{T}(s) \\
\gamma\left(s_{\gamma}\right) & =\bar{B}(s)
\end{aligned}
$$

where $s_{\alpha}$ and $s_{\gamma}$ are arc-length parameters of tangent and binormal indicatrix, respectively.

Example : Let a curve which is a slant helix be

$$
\beta(s)=\left(-\frac{3}{2} \cos \left(\frac{s}{2}\right)-\frac{1}{6} \cos \left(\frac{3 s}{2}\right),-\frac{3}{2} \sin \left(\frac{s}{2}\right)-\frac{1}{6} \sin \left(\frac{3 s}{2}\right), \sqrt{3} \cos \left(\frac{s}{2}\right)\right) .
$$

The tangent, binormal vectors, the curvature, the torsion and the Darboux vector were found in (Macit \& Düldül, 2014).

$$
\begin{aligned}
& T(s)=\left(\frac{3}{4} \sin \left(\frac{s}{2}\right)+\frac{1}{4} \sin \left(\frac{3 s}{2}\right),-\frac{3}{4} \cos \left(\frac{s}{2}\right)-\frac{1}{4} \cos \left(\frac{3 s}{2}\right),-\frac{\sqrt{3}}{2} \sin \left(\frac{s}{2}\right)\right) \\
& B(s)=\left(-\frac{1}{2} \cos \left(\frac{s}{2}\right)\left(2 \cos ^{2}\left(\frac{s}{2}\right)-3\right), \sin ^{3}\left(\frac{s}{2}\right), \frac{\sqrt{3}}{2} \cos \left(\frac{s}{2}\right)\right)
\end{aligned}
$$


and

$$
\kappa(s)=\frac{\sqrt{3}}{2} \cos \left(\frac{s}{2}\right), \quad \tau(s)=-\frac{\sqrt{3}}{2} \sin \left(\frac{s}{2}\right) .
$$

Also the W-direction curve of $\beta$ was given as:

$$
\bar{\beta}(s)=\left(-\frac{9 s}{8}-6 \sin \left(\frac{s}{2}\right)-\frac{3}{4} \sin (s)-\frac{1}{16} \sin (2 s),-\frac{1}{2} \cos (s), \frac{\sqrt{3} s}{2}\right)+\left(c_{1}, c_{2}, c_{3}\right)
$$

where $c_{1}, c_{2}, c_{3}$ are constants.

Now lets find the tangent and binormal indicatrices of this W-direction curve $\bar{\beta}$. By using these expressions above and the equations (2.3), (3.1) and (3.2), the tangent indicatrix and binormal indicatrix are obtained respectively:

$$
\alpha\left(s_{\alpha}\right)=\left(\frac{1}{2} \cos s, \frac{1}{2} \sin s, \frac{\sqrt{3}}{2}\right)
$$

and

$$
\gamma\left(s_{\gamma}\right)=\left(\frac{\sqrt{3}}{2} \cos s, \frac{\sqrt{3}}{2} \sin s,-\frac{1}{2}\right) .
$$

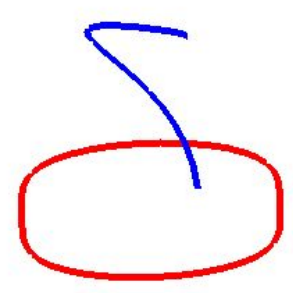

Figure 1. Red curve $=$ main curve $(\beta)$, blue curve $=$ w-direction curve of the main curve $(\bar{\beta})$

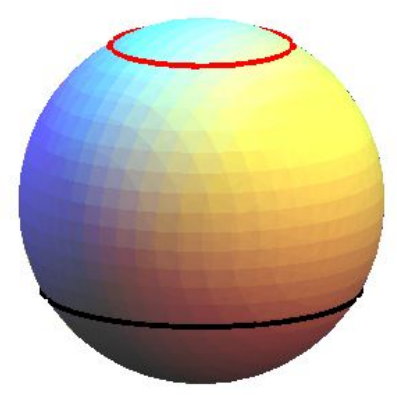

Figure 2. Red curve=tangent indicatrix $(\alpha)$, blue curve=binormal indicatrix $(\gamma)$ 
Theorem 3. Let $\beta$ be a curve with arc-length parameter s and $\bar{\beta}$ be the $W$-direction curve of $\beta$. The Frenet vector fields, curvature and torsion of the tangent indicatrix $\alpha$ of $W$-direction curve are given by

$$
\begin{aligned}
& T_{\alpha}=-\frac{1}{\sqrt{1+f^{2}}} T+\frac{f}{\sqrt{1+f^{2}}} B \\
& N_{\alpha}=-\frac{\kappa^{\prime}(f-g)}{\sqrt{\left(\kappa^{\prime}\right)^{2}(f-g)^{2}\left(1+f^{2}\right)+\kappa^{4}\left(1+f^{2}\right)^{4}}}\left(f T+\frac{\kappa^{2}\left(1+f^{2}\right)^{2}}{\kappa^{\prime}(f-g)} N+B\right) \\
& B_{\alpha}=\frac{\kappa\left(1+f^{2}\right)}{\sqrt{\left(\kappa^{\prime}\right)^{2}(f-g)^{2}+\kappa^{4}\left(1+f^{2}\right)^{3}}}\left(\tau T-\frac{\kappa^{\prime}(f-g)}{\kappa\left(1+f^{2}\right)} N+\kappa B\right) \\
& \kappa_{\alpha}=\sqrt{1+\frac{\kappa^{4}\left(1+f^{2}\right)^{3}}{\left(\kappa^{\prime}\right)^{2}(f-g)^{2}}}\left(3\left(\kappa^{\prime}\right)^{2}(1+f g)(f-g)-\kappa \kappa^{\prime \prime}(f-h)\left(1+f^{2}\right)\right) \\
& \tau_{\alpha}=\frac{\kappa^{2} \sqrt{\left(1+f^{2}\right)^{3}}}{\kappa^{\prime}(f-g)\left(\left(\kappa^{\prime}\right)^{2}(f-g)^{2}+\kappa^{4}\left(1+f^{2}\right)^{3}\right)}(t)
\end{aligned}
$$

where $f=\frac{\tau}{\kappa}, \quad g=\frac{\tau^{\prime}}{\kappa^{\prime}}$ and $h=\frac{\tau^{\prime \prime}}{\kappa^{\prime \prime}}$.

Proof. The equation of tangent indicatrix $\alpha$ is given in equation (3.1) with the arc-length parameter $s_{\alpha}$. By differentiating equation (3.1) and using Frenet formulas we get

$$
\frac{d s_{\alpha}}{d s}=\bar{\kappa}
$$

If we use the equations in (2.1) and the relation $\frac{d s_{\alpha}}{d s}=\bar{\kappa}$, we find the tangent, principal and binormal vector fields respectively as:

$$
\begin{aligned}
& T_{\alpha}=\bar{N} \\
& N_{\alpha}=-\frac{\bar{\kappa}}{\sqrt{\bar{\kappa}^{2}+\bar{\tau}^{2}}} \bar{T}+\frac{\bar{\tau}}{\sqrt{\bar{\kappa}^{2}+\bar{\tau}^{2}}} \bar{B} \\
& B_{\alpha}=\frac{\bar{\tau}}{\sqrt{\bar{\kappa}^{2}+\bar{\tau}^{2}}} \bar{T}+\frac{\bar{\kappa}}{\sqrt{\bar{\kappa}^{2}+\bar{\tau}^{2}}} \bar{B}
\end{aligned}
$$

By writing the relations (2.3) in the last equations, we have:

$$
\begin{aligned}
& T_{\alpha}=-\frac{\kappa}{\sqrt{\kappa^{2}+\tau^{2}}} T+\frac{\tau}{\sqrt{\kappa^{2}+\tau^{2}}} B \\
& N_{\alpha}=-\frac{\tau \kappa^{\prime}-\tau^{\prime} \kappa}{\sqrt{\left(\tau \kappa^{\prime}-\tau^{\prime} \kappa\right)^{2}+\left(\kappa^{2}+\tau^{2}\right)^{3}}}\left(\frac{\tau}{\sqrt{\kappa^{2}+\tau^{2}}} T+\frac{\sqrt{\left(\kappa^{2}+\tau^{2}\right)^{3}}}{\tau \kappa^{\prime}-\tau^{\prime} \kappa} N+\frac{\kappa}{\sqrt{\kappa^{2}+\tau^{2}}} B\right) \\
& B_{\alpha}=\frac{\kappa^{2}+\tau^{2}}{\sqrt{\left(\tau \kappa^{\prime}-\tau^{\prime} \kappa\right)^{2}+\left(\kappa^{2}+\tau^{2}\right)^{3}}}\left(\tau T-\frac{\tau \kappa^{\prime}-\tau^{\prime} \kappa}{\kappa^{2}+\tau^{2}} N+\kappa B\right) .
\end{aligned}
$$

In the last equations assuming $f=\frac{\tau}{\kappa}, g=\frac{\tau^{\prime}}{\kappa^{\prime}}$ and $h=\frac{\tau^{\prime \prime}}{\kappa^{\prime \prime}}$ and arranging the expressions, we obtain the tangent, principal and binormal vector fields of the tangent indicatrix $\alpha$ of the W-direction curve $\bar{\beta}$, with respect to the main curve $\beta$.

Also the curvature and torsion of the tangent indicatrix $\alpha$ are found as:

$$
\begin{aligned}
& \kappa_{\alpha}=\frac{\sqrt{\bar{\kappa}^{2}+\bar{\tau}^{2}}}{\bar{\kappa}} \\
& \tau_{\alpha}=\sqrt{\frac{\left(\bar{\tau}^{\prime} \bar{\kappa}-\overline{\tau \kappa}^{\prime}\right)^{2}+\left(\bar{\kappa}^{\prime} \frac{\bar{\tau}^{2}}{\bar{\kappa}}-\bar{\tau} \bar{\tau}^{\prime}\right)^{2}}{\left(\bar{\kappa}^{2}+\bar{\tau}^{2}\right)^{3}}} .
\end{aligned}
$$


Again by using the relations (2.3) in equations (3.3) and (3.4), we get:

$$
\begin{aligned}
\kappa_{\alpha} & =\frac{\sqrt{\left(\tau \kappa^{\prime}-\tau^{\prime} \kappa\right)^{2}+\left(\kappa^{2}+\tau^{2}\right)^{3}}}{\tau \kappa^{\prime}-\tau^{\prime} \kappa} \\
\tau_{\alpha} & =\frac{\sqrt{\left(\kappa^{2}+\tau^{2}\right)^{3}}}{\left(\tau \kappa^{\prime}-\tau^{\prime} \kappa\right)\left(\left(\tau \kappa^{\prime}-\tau^{\prime} \kappa\right)^{2}+\left(\kappa^{2}+\tau^{2}\right)^{3}\right)}\left(\begin{array}{c}
3\left(\kappa \kappa^{\prime}+\tau \tau^{\prime}\right)\left(\tau \kappa^{\prime}-\tau^{\prime} \kappa\right) \\
-\left(\tau \kappa^{\prime \prime}-\tau^{\prime \prime} \kappa\right)\left(\kappa^{2}+\tau^{2}\right)
\end{array}\right) .
\end{aligned}
$$

Taking $f, g$ and $h$ in the last equations, we reach the result.

Theorem 4. If any curve $\beta$ with arc-length parameter $s$ is slant helix, then the tangent indicatrix of W-direction curve of $\beta$ is a planar curve (namely, a circle or part of a circle).

Proof. By Theorem 6 in (Macit \& Düldül, 2014), if the geodesic curvature of the principal image of the principal normal indicatrix of the curve $\beta$ is $\delta$, then $\frac{\bar{\kappa}}{\bar{\tau}}=\delta$ where $\bar{\kappa}$ and $\bar{\tau}$ are curvature and torsion of the W-direction curve of $\beta$.

By using the equation (3.4) and $\frac{\bar{\kappa}}{\bar{\tau}}=\delta$, the torsion of the tangent indicatrix $\alpha$ is found as:

$$
\tau_{\alpha}=\frac{\delta^{\prime}}{\bar{\tau} \delta\left(1+\delta^{2}\right)} .
$$

If the curve $\beta$ is slant helix, then $\delta^{\prime}=0$. So by the last equation $\tau_{\alpha}=0$ which means the tangent indicatrix $\alpha$ is planar. The planar curves on the sphere are circles or part of circles.

Remark 2. From now on, the equations $f=\frac{\tau}{\kappa}, \quad g=\frac{\tau^{\prime}}{\kappa^{\prime}}, h=\frac{\tau^{\prime \prime}}{\kappa^{\prime \prime}}, \delta=\frac{\bar{\kappa}}{\bar{\tau}}$ and $\kappa^{\prime}(f-g)=-\delta \kappa^{2}\left(1+f^{2}\right)^{3 / 2}$ will be used in the calculations.

Theorem 5. The tangent indicatrix $\alpha$ of the W-direction curve is a general helix if and only if the following equation is satisfied:

$$
A^{\prime}-A\left(\frac{3 \kappa^{\prime}}{\kappa}+\frac{4 f f^{\prime}}{1+f^{2}}+\frac{3 \delta \delta^{\prime}}{1+\delta^{2}}\right)=0
$$

where $A=\kappa^{\prime \prime}(f-h)+3 \kappa \kappa^{\prime} \sqrt{1+f^{2}}(1+f g) \delta$.

Proof. If we take ratio of the torsion and curvature of the tangent indicatrix which are in Theorem 3 , use the relation $\kappa^{\prime}(f-g)=-\delta \kappa^{2}\left(1+f^{2}\right)^{3 / 2}$ and make some appropriate calculations, we have:

$$
\frac{\tau_{\alpha}}{\kappa_{\alpha}}=-\frac{A}{\kappa^{3}\left(1+f^{2}\right)^{2}\left(1+\delta^{2}\right)^{3 / 2}} .
$$

By differentiating the equation (3.5), we find that:

$$
\left(\frac{\tau_{\alpha}}{\kappa_{\alpha}}\right)^{\prime}=-\frac{A^{\prime}-A\left(\frac{3 \kappa^{\prime}}{\kappa}+\frac{4 f f^{\prime}}{1+f^{2}}+\frac{3 \delta \delta^{\prime}}{1+\delta^{2}}\right)}{\kappa^{3}\left(1+f^{2}\right)^{2}\left(1+\delta^{2}\right)^{3 / 2}} .
$$

If the numerator of the last fraction is zero, then $\left(\frac{\tau_{\alpha}}{\kappa_{\alpha}}\right)^{\prime}=0$. Since the harmonic curvature of the curve $\alpha$ is constant, then it is a general helix.

Corollary 1. If the curve $\beta$ is a general helix and and the equation $A^{\prime}-3 A \frac{\kappa^{\prime}}{\kappa}=0$ is satisfied, then the tangent indicatrix of the $W$-direction curve $\bar{\beta}$ is a general helix.

Proof. If $\beta$ is a general helix, then $f$ is constant and also $f^{\prime}=0$. Since $f^{\prime}=0$, then $\delta=0$. For the derivative, we find:

$$
\left(\frac{\tau_{\alpha}}{\kappa_{\alpha}}\right)^{\prime}=-\frac{A^{\prime}-A \frac{3 \kappa^{\prime}}{\kappa}}{\kappa^{3}\left(1+f^{2}\right)^{2}\left(1+\delta^{2}\right)^{3 / 2}} .
$$

If the numerator of this fraction is zero, then we reach the result clearly.

Theorem 6. The tangent indicatrix $\alpha$ of the W-direction curve is a slant helix if and only if the following equation is satisfied:

$$
\delta^{\prime}\left(1+4 \delta^{2}\right) \frac{X}{Y}+\delta\left(1+\delta^{2}\right)\left(\frac{X}{Y}\right)^{\prime}=0
$$

where $\delta=\frac{\bar{\kappa}}{\bar{\tau}}, X=\left(1+\delta^{2}\right) \bar{\tau}\left(\delta^{\prime \prime} \bar{\tau}-\delta^{\prime} \bar{\tau}^{\prime}\right)-3 \delta\left(\delta^{\prime}\right)^{2} \bar{\tau}^{2}$ and $Y=\left(\bar{\tau}^{2}\left(1+\delta^{2}\right)^{3}+\left(\delta^{\prime}\right)^{2}\right)^{3 / 2}$. 
Proof. From the equation (1.1), the geodesic curvature of the principal image of the principal normal indicatrix of the tangent indicatrix $\alpha$ is given by

$$
\delta_{\alpha}=\frac{\kappa_{\alpha}^{2}}{\left(\kappa_{\alpha}^{2}+\tau_{\alpha}^{2}\right)^{\frac{3}{2}}} \cdot\left(\frac{\tau_{\alpha}}{\kappa_{\alpha}}\right)^{\prime} .
$$

We take into account that $\bar{\tau}^{\prime} \bar{\kappa}-\overline{\tau \kappa}^{\prime}=-\bar{\tau}^{2} \delta^{\prime}, \quad \bar{\kappa}^{\prime} \frac{\bar{\tau}^{2}}{\bar{\kappa}}-\bar{\tau}^{\prime}=\bar{\tau}^{2} \frac{\delta^{\prime}}{\delta}$ and $\bar{\kappa}^{2}+\bar{\tau}^{2}=\bar{\tau}^{2}\left(1+\delta^{2}\right)$, then put the equations (3.3) and (3.4) in (3.6), we clearly find

$$
\delta_{\alpha}=\frac{1+\delta^{2}}{\delta^{2}}\left(\frac{\delta^{2}\left(1+\delta^{2}\right)^{2} \bar{\tau}^{2}}{\left(1+\delta^{2}\right)^{3} \bar{\tau}^{2}+\left(\delta^{\prime}\right)^{2}}\right)^{3 / 2}\left(\frac{\delta^{\prime}}{\bar{\tau}\left(1+\delta^{2}\right)^{3 / 2}}\right)^{\prime} .
$$

After some calculations we have

$$
\delta_{\alpha}=\frac{\delta\left(1+\delta^{2}\right)^{3 / 2} X}{Y}
$$

where $X=\left(1+\delta^{2}\right) \bar{\tau}\left(\delta^{\prime \prime} \bar{\tau}-\delta^{\prime} \bar{\tau}^{\prime}\right)-3 \delta\left(\delta^{\prime}\right)^{2} \bar{\tau}^{2}$ and $Y=\left(\bar{\tau}^{2}\left(1+\delta^{2}\right)^{3}+\left(\delta^{\prime}\right)^{2}\right)^{3 / 2}$.

By differentiating the equation (3.7), we obtain

$$
\left(\delta_{\alpha}\right)^{\prime}=\left(1+\delta^{2}\right)^{1 / 2}\left(\delta^{\prime}\left(1+4 \delta^{2}\right) \frac{X}{Y}+\delta\left(1+\delta^{2}\right)\left(\frac{X}{Y}\right)^{\prime}\right) .
$$

$\left(\delta_{\alpha}\right)^{\prime}=0$ if and only if $\delta^{\prime}\left(1+4 \delta^{2}\right) \frac{X}{Y}+\delta\left(1+\delta^{2}\right)\left(\frac{X}{Y}\right)^{\prime}=0$.

So by taking into consideration the equation (1.1), the proof is completed.

Theorem 7. Let $\beta$ be a curve with arc-length parameter s and $\bar{\beta}$ be the W-direction curve of $\beta$. The Frenet vector fields, curvature and torsion of the binormal indicatrix $\gamma$ of W-direction curve are given by

$$
\begin{aligned}
T_{\gamma} & =\frac{1}{\sqrt{1+f^{2}}} T-\frac{f}{\sqrt{1+f^{2}}} B \\
N_{\gamma} & =\frac{\kappa^{\prime}(f-g)}{\sqrt{\left(\kappa^{\prime}\right)^{2}(f-g)^{2}\left(1+f^{2}\right)+\kappa^{4}\left(1+f^{2}\right)^{4}}}\left(f T+\frac{\kappa^{2}\left(1+f^{2}\right)^{2}}{\kappa^{\prime}(f-g)} N+B\right) \\
B_{\gamma} & =\frac{\kappa\left(1+f^{2}\right)}{\sqrt{\left(\kappa^{\prime}\right)^{2}(f-g)^{2}+\kappa^{4}\left(1+f^{2}\right)^{3}}}\left(\tau T-\frac{\kappa^{\prime}(f-g)}{\kappa\left(1+f^{2}\right)} N+\kappa B\right) \\
\kappa_{\gamma} & =\sqrt{1+\frac{\left(\kappa^{\prime}\right)^{2}(f-g)^{2}}{\kappa^{4}\left(1+f^{2}\right)^{3}}} \\
\tau_{\gamma} & =\frac{1}{\left(\kappa^{\prime}\right)^{2}(f-g)^{2}+\kappa^{4}\left(1+f^{2}\right)^{3}}\left(3\left(\kappa^{\prime}\right)^{2}(1+f g)(f-g)-\kappa \kappa^{\prime \prime}(f-h)\left(1+f^{2}\right)\right)
\end{aligned}
$$

where $f=\frac{\tau}{\kappa}, \quad g=\frac{\tau^{\prime}}{\kappa^{\prime}}$ and $h=\frac{\tau^{\prime \prime}}{\kappa^{\prime \prime}}$.

Proof. The equation of binormal indicatrix $\gamma$ is given in equation (3.2) with the arc-length parameter $s_{\gamma}$. By differentiating equation (3.2) and using Frenet formulas we get

$$
\frac{d s_{\gamma}}{d s}=|\bar{\tau}|
$$

Here we assume $\bar{\tau}>0$. If $\bar{\tau}<0$, the Frenet vector fields have other signs.

If we use the equations in (2.1) and the relation $\frac{d s_{\gamma}}{d s}=\bar{\tau}$, we find the tangent, principal and binormal vector fields respectively as:

$$
\begin{aligned}
& T_{\gamma}=-\bar{N} \\
& N_{\gamma}=\frac{\bar{\kappa}}{\sqrt{\bar{\kappa}^{2}+\bar{\tau}^{2}}} \bar{T}-\frac{\bar{\tau}}{\sqrt{\bar{\kappa}^{2}+\bar{\tau}^{2}}} \bar{B} \\
& B_{\gamma}=\frac{\bar{\tau}}{\sqrt{\bar{\kappa}^{2}+\bar{\tau}^{2}}} \bar{T}+\frac{\bar{\kappa}}{\sqrt{\bar{\kappa}^{2}+\bar{\tau}^{2}}} \bar{B} .
\end{aligned}
$$


By writing the relations (2.3) in the last equations, we have:

$$
\begin{aligned}
& T_{\gamma}=\frac{\kappa}{\sqrt{\kappa^{2}+\tau^{2}}} T-\frac{\tau}{\sqrt{\kappa^{2}+\tau^{2}}} B \\
& N_{\gamma}=\frac{\tau \kappa^{\prime}-\tau^{\prime} \kappa}{\sqrt{\left(\tau \kappa^{\prime}-\tau^{\prime} \kappa\right)^{2}+\left(\kappa^{2}+\tau^{2}\right)^{3}}}\left(\frac{\tau}{\sqrt{\kappa^{2}+\tau^{2}}} T+\frac{\sqrt{\left(\kappa^{2}+\tau^{2}\right)^{3}}}{\tau \kappa^{\prime}-\tau^{\prime} \kappa} N+\frac{\kappa}{\sqrt{\kappa^{2}+\tau^{2}}} B\right) \\
& B_{\gamma}=\frac{\kappa^{2}+\tau^{2}}{\sqrt{\left(\tau \kappa^{\prime}-\tau^{\prime} \kappa\right)^{2}+\left(\kappa^{2}+\tau^{2}\right)^{3}}}\left(\tau T-\frac{\tau \kappa^{\prime}-\tau^{\prime} \kappa}{\kappa^{2}+\tau^{2}} N+\kappa B\right) .
\end{aligned}
$$

In the last equations assuming $f=\frac{\tau}{\kappa}, g=\frac{\tau^{\prime}}{\kappa^{\prime}}$ and $h=\frac{\tau^{\prime \prime}}{\kappa^{\prime \prime}}$, arranging the expressions, we obtain the tangent, principal and binormal vector fields of the binormal indicatrix $\gamma$ of the W-direction curve $\bar{\beta}$, with respect to the main curve $\beta$.

Also the curvature and torsion of the binormal indicatrix $\gamma$ by taking into account that $\frac{d s_{\gamma}}{d s}=\bar{\tau}$ are found as:

$$
\begin{aligned}
& \kappa_{\gamma}=\frac{\sqrt{\bar{\kappa}^{2}+\bar{\tau}^{2}}}{\bar{\tau}} \\
& \tau_{\gamma}=\sqrt{\frac{\left(\overline{\tau \kappa}-\bar{\tau}^{\prime} \bar{\kappa}\right)^{2}+\left(\bar{\tau}^{\prime} \frac{\bar{K}^{2}}{\overline{\bar{\tau}}}-\overline{K \kappa}^{\prime}\right)^{2}}{\left(\bar{\kappa}^{2}+\bar{\tau}^{2}\right)^{3}}} .
\end{aligned}
$$

Again by using the relations (2.3) in equations (3.8) and (3.9), we get:

$$
\begin{aligned}
& \kappa_{\gamma}=\frac{\sqrt{\left(\tau \kappa^{\prime}-\tau^{\prime} \kappa\right)^{2}+\left(\kappa^{2}+\tau^{2}\right)^{3}}}{\sqrt{\left(\kappa^{2}+\tau^{2}\right)^{3}}} \\
& \tau_{\gamma}=\frac{1}{\left(\tau \kappa^{\prime}-\tau^{\prime} \kappa\right)^{2}+\left(\kappa^{2}+\tau^{2}\right)^{3}}\left(\begin{array}{c}
3\left(\kappa \kappa^{\prime}+\tau \tau^{\prime}\right)\left(\tau \kappa^{\prime}-\tau^{\prime} \kappa\right) \\
-\left(\tau \kappa^{\prime \prime}-\tau^{\prime \prime} \kappa\right)\left(\kappa^{2}+\tau^{2}\right)
\end{array}\right) .
\end{aligned}
$$

Taking $f, g$ and $h$ in the last equations, we reach the result.

Corollary 2. The tangent indicatrix and the binormal indicatrix of a W-direction curve are Bertrand mate curves.

Proof. Since the relation between the principal normal vector fields of the tangent indicatrix and binormal indicatrix is:

$$
N_{\alpha}=-N_{\gamma},
$$

they are linearly dependent. Thus the result is apparent.

Theorem 8. If any curve $\beta$ with arc-length parameter s is slant helix, then the binormal indicatrix of $W$-direction curve of $\beta$ is a planar curve (namely, a circle or part of a circle).

Proof. By using the equation (3.9) and $\frac{\overline{\underline{K}}}{\bar{\tau}}=\delta$, the torsion of the binormal indicatrix $\gamma$ is found as:

$$
\tau_{\gamma}=\frac{\delta^{\prime}}{\bar{\tau}\left(1+\delta^{2}\right)} .
$$

If the curve $\beta$ is slant helix, then $\delta^{\prime}=0$. So by the last equation $\tau_{\gamma}=0$ which means the binormal indicatrix $\gamma$ is planar. The planar curves on the sphere are circles or part of circles.

Theorem 9. The binormal indicatrix $\gamma$ of the $W$-direction curve is a general helix if and only if the following equation is satisfied:

$$
A^{\prime}-A\left(\frac{3 \kappa^{\prime}}{\kappa}+\frac{4 f f^{\prime}}{1+f^{2}}+\frac{3 \delta \delta^{\prime}}{1+\delta^{2}}\right)=0
$$

where $A=\kappa^{\prime \prime}(f-h)+3 \kappa \kappa^{\prime} \sqrt{1+f^{2}}(1+f g) \delta$. 
Proof. If we take ratio of the torsion and curvature of the binormal indicatrix which are in Theorem 7 , use the relation $\kappa^{\prime}(f-g)=-\delta \kappa^{2}\left(1+f^{2}\right)^{3 / 2}$ and make some appropriate calculations, we have:

$$
\begin{aligned}
\frac{\tau_{\gamma}}{\kappa_{\gamma}} & =\frac{3\left(\kappa^{\prime}\right)^{2}(1+f g)(f-g)-\kappa \kappa^{\prime \prime}(f-h)\left(1+f^{2}\right)}{\left(\kappa^{\prime}\right)^{2}(f-g)^{2}+\kappa^{4}\left(1+f^{2}\right)^{3}} \cdot \frac{\kappa^{2}\left(1+f^{2}\right)^{3 / 2}}{\sqrt{\kappa^{4}\left(1+f^{2}\right)^{3}+\left(\kappa^{\prime}\right)^{2}(f-g)^{2}}} \\
& =\frac{3\left(\kappa^{\prime}\right)^{2}(1+f g)(f-g) \kappa^{2}\left(1+f^{2}\right)^{3 / 2}-\kappa^{3} \kappa^{\prime \prime}(f-h)\left(1+f^{2}\right)^{5 / 2}}{\left(\kappa^{4}\left(1+f^{2}\right)^{3}+\left(\kappa^{\prime}\right)^{2}(f-g)^{2}\right)^{3 / 2}} \\
& =\frac{-3 \delta \kappa^{4} \kappa^{\prime}(1+f g)\left(1+f^{2}\right)^{3}-\kappa^{3} \kappa^{\prime \prime}(f-h)\left(1+f^{2}\right)^{5 / 2}}{\left(\kappa^{4}\left(1+f^{2}\right)^{3}\left(1+\delta^{2}\right)\right)^{3 / 2}} \\
& =\frac{\kappa^{3}\left(1+f^{2}\right)^{5 / 2}\left(-3 \delta \kappa \kappa^{\prime}(1+f g)\left(1+f^{2}\right)^{1 / 2}-\kappa^{\prime \prime}(f-h)\right)}{\kappa^{6}\left(1+f^{2}\right)^{9 / 2}\left(1+\delta^{2}\right)^{3 / 2}} .
\end{aligned}
$$

So lastly we find:

$$
\frac{\tau_{\gamma}}{\kappa_{\gamma}}=-\frac{A}{\kappa^{3}\left(1+f^{2}\right)^{2}\left(1+\delta^{2}\right)^{3 / 2}} .
$$

By differentiating the equation (3.10), we find that:

$$
\left(\frac{\tau_{\gamma}}{\kappa_{\gamma}}\right)^{\prime}=-\frac{A^{\prime}-A\left(\frac{3 \kappa^{\prime}}{\kappa}+\frac{4 f f^{\prime}}{1+f^{2}}+\frac{3 \delta \delta^{\prime}}{1+\delta^{2}}\right)}{\kappa^{3}\left(1+f^{2}\right)^{2}\left(1+\delta^{2}\right)^{3 / 2}} .
$$

If the numerator of the last fraction is zero, then $\left(\frac{\tau_{\alpha}}{\kappa_{\alpha}}\right)^{\prime}=0$. Since the harmonic curvature of the curve $\gamma$ is constant, it is a general helix.

Corollary 3. Let $\beta$ be a curve with arc-length parameter $s$ and $\bar{\beta}$ be the $W$-direction curve of $\beta$. The tangent indicatrix of $\bar{\beta}$ is a general helix if and only if the binormal indicatrix of $\bar{\beta}$ is general helix.

Proof. By the equations (3.5) and (3.10), the result is clear.

Theorem 10. The binormal indicatrix $\gamma$ of the W-direction curve is a slant helix if and only if the following equation is satisfied:

$$
3 \delta \delta^{\prime} \frac{X}{Y}+\left(1+\delta^{2}\right)\left(\frac{X}{Y}\right)^{\prime}=0
$$

where $\delta=\frac{\bar{\kappa}}{\bar{\tau}}, X=\left(1+\delta^{2}\right) \bar{\tau}\left(\delta^{\prime \prime} \bar{\tau}-\delta^{\prime} \bar{\tau}^{\prime}\right)-3 \delta\left(\delta^{\prime}\right)^{2} \bar{\tau}^{2}$ and $Y=\left(\bar{\tau}^{2}\left(1+\delta^{2}\right)^{3}+\left(\delta^{\prime}\right)^{2}\right)^{3 / 2}$.

Proof. From the equation (1.1), the geodesic curvature of the principal image of the principal normal indicatrix of the binormal indicatrix $\gamma$ is given by

$$
\delta_{\gamma}=\frac{\kappa_{\gamma}^{2}}{\left(\kappa_{\gamma}^{2}+\tau_{\gamma}^{2}\right)^{\frac{3}{2}}} \cdot\left(\frac{\tau_{\gamma}}{\kappa_{\gamma}}\right)^{\prime} .
$$

We take into account that $\bar{\tau}^{\prime} \bar{\kappa}-\overline{\tau \kappa}^{\prime}=-\bar{\tau}^{2} \delta^{\prime}, \quad \bar{\kappa}^{\prime} \frac{\bar{\tau}^{2}}{\bar{\kappa}}-\bar{\tau}^{\prime}=\bar{\tau}^{2} \frac{\delta^{\prime}}{\delta}$ and $\bar{\kappa}^{2}+\bar{\tau}^{2}=\bar{\tau}^{2}\left(1+\delta^{2}\right)$, then put the equations (3.8) and (3.9) in (3.11), we clearly find

$$
\delta_{\gamma}=\frac{\bar{\tau}^{3}\left(1+\delta^{2}\right)^{4}}{\left(\left(1+\delta^{2}\right)^{3} \bar{\tau}^{2}+\left(\delta^{\prime}\right)^{2}\right)^{3 / 2}}\left(\frac{\delta^{\prime}}{\bar{\tau}\left(1+\delta^{2}\right)^{3 / 2}}\right)^{\prime} .
$$

After some calculations we have

$$
\delta_{\gamma}=\frac{\left(1+\delta^{2}\right)^{3 / 2} X}{Y}
$$

where $X=\left(1+\delta^{2}\right) \bar{\tau}\left(\delta^{\prime \prime} \bar{\tau}-\delta^{\prime} \bar{\tau}^{\prime}\right)-3 \delta\left(\delta^{\prime}\right)^{2} \bar{\tau}^{2}$ and $Y=\left(\bar{\tau}^{2}\left(1+\delta^{2}\right)^{3}+\left(\delta^{\prime}\right)^{2}\right)^{3 / 2}$.

By differentiating the equation (3.12), we obtain

$$
\left(\delta_{\gamma}\right)^{\prime}=\left(1+\delta^{2}\right)^{1 / 2}\left(3 \delta \delta^{\prime} \frac{X}{Y}+\left(1+\delta^{2}\right)\left(\frac{X}{Y}\right)^{\prime}\right) .
$$

$\left(\delta_{\gamma}\right)^{\prime}=0$ if and only if $3 \delta \delta^{\prime} \frac{X}{Y}+\left(1+\delta^{2}\right)\left(\frac{X}{Y}\right)^{\prime}=0$.

So by taking into consideration the equation (1.1), the proof is completed. 
Corollary 4. The relation between the geodesic curvatures of the principal images of the principal normal indicatrices of the tangent indicatrix, binormal indicatrix and the curve $\beta$ is:

$$
\delta_{\alpha}=\delta . \delta_{\gamma} .
$$

Proof. By the equations (3.7) and (3.12), we have

$$
\delta_{\alpha}=\delta . \delta_{\gamma}
$$

where $\delta=\frac{\bar{\kappa}}{\bar{\tau}}$.

Remark 3. The example given at the top of the Section 3 is an application of the theorems 4 and 8.

\section{References}

Choi, J. H., \& Kim, Y. H. (2012). Associated curves of a Frenet curve and their applications. Applied Mathematics and Computation., 218, 9116-9124. https://doi.org/10.1016/j.amc.2012.02.064

Choi, J. H., Kim, Y. H., \& Ali, A. T. (2012). Some associated curves of Frenet non-lightlike curves in $\mathbb{E}_{1}^{3}$. Journal of Mathematical Analysis and Applications, 394, 712-723. https://doi.org/10.1016/j.jmaa.2012.04.063

Gray, A. (1993). Modern differential geometry of curves and surfaces with mathematica (2nd ed.). Boca Raton, FL: Crc Press.

Izumiya, S., \& Takeuchi, N. (2003). Special curves and ruled surfaces. Beitrage zur Alg und Geo Contributions to Alg and Geo, 44(1), 203-212.

Izumiya, S., \& Takeuchi, N. (2004). New special curves and developable surfaces. Turkish Journal of Mathematics, 28, 153-163.

Körpınar, T., Sarıaydın, M. T., \& Turhan, E. (2013). Associated curves according to Bishop frame in Euclidean 3-space. Advanced Modelling and Optimization, 15, 713-717.

Kula, L., \& Yaylı, Y. (2005). On slant helix and its spherical indicatrix. Applied Mathematics and Computation, 169, 600-607. https://doi.org/10.1016/j.amc.2004.09.078

Kula, L., Ekmekci, N., Yaylı, Y., \& İlarslan, K. (2010). Characterizations of slant helices in Euclidean 3-space. Turkish Journal of Mathematics, 34, 261-273. https://doi.org/10.3906/ mat-0809-17

Kruppa, E. (1957). Analytische und Constructive Differential Geometrie. Wien: Springer Verlag.

Macit, N., \& Düldül, M. (2014). Some new associated curves of a Frenet curve in $\mathbb{E}^{3}$ and $\mathbb{E}^{4}$. Turkish Journal of Mathematics, 38, 1023-1037. https://doi.org/10.3906/mat-1401-85

O’Neill, B. (2006). Elementary Differential Geometry(2nd ed.). Academic Press, Burlington, USA.

Struik, D. J. (1957). Lectures On Classical Differential Geometry (2nd ed.). Addision-Wesley Publishing Company, Inc, USA.

Tunçer, Y., \& Ünal, S. (2012). New representations of Bertrand pairs in Euclidean 3-space. Applied Mathematics and Computation, 219(4), 1833-1842. https://doi.org/10.1016/j.amc.2012.08.023

\section{Copyrights}

Copyright for this article is retained by the author(s), with first publication rights granted to the journal.

This is an open-access article distributed under the terms and conditions of the Creative Commons Attribution license (http://creativecommons.org/licenses/by/4.0/). 